\title{
One-Step Microfluidic Fabrication of Multi-Responsive Liposomes for Targeted Delivery of Doxorubicin Synergism with Photothermal Effect
}

\author{
Songwei $\operatorname{Lv} \mathbb{D}^{1, *}$ \\ Ran Jing ${ }^{2, *}$ \\ Xiaowu $\mathrm{Liu}^{3,4}$ \\ Honglei Shi ${ }^{3,4}$ \\ Yunfeng $\mathrm{Shi}^{3,4}$ \\ Xugang Wang ${ }^{3,4}$ \\ Xiubo Zhao ${ }^{1,5}$ \\ Kai $\mathrm{CaO}^{3,4}$ \\ Zhong $\mathrm{Lv}^{3,4}$
}

'School of Pharmacy, Changzhou University, Changzhou, 213164, People's Republic of China; ${ }^{2}$ Division of Nephrology, The Affiliated Changzhou NO. 2 People's Hospital of Nanjing Medical University, Changzhou, 213164, People's Republic of China; ${ }^{3}$ Department of Urology, Wujin Hospital Affiliated with Jiangsu University, Changzhou, 213164, People's Republic of China; ${ }^{4}$ Department of Urology, The Wujin Clinical College of Xuzhou Medical University, Changzhou, 213164, People's Republic of China; ${ }^{5}$ Department of Chemical and Biological Engineering, University of Sheffield, Sheffield, SI 3JD, UK

*These authors contributed equally to this work

\begin{abstract}
Introduction: Cancer of the bladder is one of the most common and life-threatening. Compared with traditional delivery methods, intravesical administration reduces the amount of drugs required, increases the amount of drugs reaching the lesion site, and minimizes systemic exposure to therapeutic agents. To overcome the limitations of urinary voiding, low urothelium permeability, and intermittent catheterization for large dilution and irrigation of drugs in the bladder, magnetic and photothermal-responsive folate receptor-targeted thermal liposomes (FA-TMLs) were designed for the targeted delivery of doxorubicin (DOX) to bladder cancer cells.
\end{abstract}

Methods: Through a microfluidic mixer chip, the magnetic nanoparticles (MNPs), gold nanorods (GNRs) and DOX were encapsulated in folate-modified thermosensitive liposomes to form FA-TMLs@MNPs-GNRs-DOX. DLS, TEM, DSC, and magnetic hysteresis loop were used to characterize the construction of FA-TMLs@MNPs-GNRs-DOX.

Results: FA-TMLs@MNPs-GNRs-DOX had a size of about $230 \mathrm{~nm}$ and exhibited superparamagnetic properties with the saturation magnetization of $20 \mathrm{emu} / \mathrm{g}$. The DOX loading capacity was as high as $0.57 \mathrm{mg} / \mathrm{mL}$. Additionally, drug release of the FA-TMLs@MNPsGNRs-DOX could be controlled by temperature change through the photothermal effect. A $980 \mathrm{~nm}$ laser beam was selectively irradiated on the FA-TMLs@MNPs-GNRs-DOX to trigger the structural changes of the FA-TMLs, and an average of $95 \%$ of the drug was released after 3 hours. The results of cell uptake experiments reveal indicated that FATMLs@MNPs-GNRs-DOX were able to specifically bind folate-receptor-positive cells and exhibited toxicity to bladder tumor cells.

Conclusion: The present results suggest FA-TMLs@MNPs-GNRs-DOX have a promising multifunctional response and can act as an ideal multifunctional drug delivery system (DDS) for the treatment of bladder tumors.

Keywords: bladder cancer, drug delivery, magnetic response, thermo-sensitive liposomes, folate-targeted, photothermal effect

\section{Introduction}

As one of the most common cancers, bladder cancer (BC) is among the leading causes of high mortality from cancers of the urinary system. Globally, 430,000 people are diagnosed with $\mathrm{BC}$ every year. ${ }^{1,2}$ Approximately $75 \%$ of newly diagnosed patients have non-muscular invasive bladder cancer (NMIBC), and $25 \%$ have muscular invasive bladder cancer (MIBC) or metastatic disease. ${ }^{3,4}$ Intravesical drug
Correspondence: Kai Cao; Zhong Lv Email caokai900I@I26.com; Izabc0105@I63.com 
delivery (IDD) is a direct administration of therapeutic agents into the bladder via insertion of a urethral catheter. The standard treatment for NMIBC is transurethral resection of the bladder tumor and postoperative perfusion therapy. ${ }^{4-6}$ Intravesical perfusion therapy is a local strategy and an effective method to prevent recurrence and progression of $\mathrm{BC}$ after transurethral resection of bladder tumor. Compared with traditional delivery systems, intravesical administration reduces the amount of drugs required, increases the amount of drugs reaching the lesion site, and minimizes systemic exposure to the therapeutic agent. $^{6-8}$ Despite the significant progress made over the past few decades in the IDD field, several intrinsic limitations remain, including the substantial dilution of chemotherapy agents and wash out due to urinary voiding, low permeability of the urothelium, and intermittent catheterization. As such, there are still several critical challenges that need to be addressed to improve the targeting effect and drug concentration in intravesical treatment. ${ }^{5,6,9-11}$

Nanocarrier-based chemotherapy has been extensively applied to improve traditional cancer chemotherapy strategies. ${ }^{12-14}$ However, due to the lack of specific targeting, there are difficulties in accumulating enough chemotherapy agents at the tumor site. The ideal targeted drug delivery systems (DDSs) should be able to increase the solubility of the chemotherapy agents, protect from degradation, extend the circulation time of the drugs in the bloodstream, and deliver the drugs to the targeted tissues. ${ }^{15-17}$ In addition, DDSs should also have high drug loading, a controllable release capacity and biological safety ${ }^{18,19}$ In an effort to achieve the ideal DDS, researchers have proposed a variety of strategies to better target the nanocarriers to tumor cells in bladder tissue. ${ }^{9,20-23}$ Among said strategies, active targeting DDSs are the most promising, through which drugs are delivered to cancer tissue via linked ligands or antibodies recognizing the specific receptors on the surface of tumor cells. ${ }^{24-26}$ The folate receptor is a high glucosyl phosphatidyl inositol anchor protein on the surface of primary and metastatic tumor cells, including bladder cancer cells, but almost no folate receptor is presented on the surface of normal cells. ${ }^{27-29}$ However, DDSs need to be firstly directed to tumor sites in the before recognizing cell surface receptors. Thus, through external targeting strategies such as magnetic fields, drug carriers can be effectively accumulated to tumor tissues, thereby improving the efficiency of drug delivery. ${ }^{30-35}$ Prior research has evidenced that, under the guidance of external magnetic fields, magnetic liposomes formed by embedding ferromagnetic nanoparticles in pegylated liposomes have targeted accumulation in the desired region of interest (ROI). Through the use of external magnetic fields, magnetic liposomes have a wide range of functions and can provide a platform for active and passive targeted drug delivery. Moreover, superparamagnetic nanoparticles can be used as magnetic resonance contrast agents for realtime, targeted monitoring of drug delivery and therapeutic effects through magnetic resonance imaging (MRI).

Intelligent drug delivery systems based on nanoparticles, which can respond to a variety of exogenous or endogenous stimuli to deliver sustained drug release, are particularly needed. Near-infrared (NIR) light is a favorable exogenous stimulus for intelligent drug delivery systems. NIR mediated photothermal therapy (PTT) is also regarded as a promising method for cancer treatment owing to the advantages of deep tissue penetration, targeted selectivity, improved therapeutic effect and minimal damage to surrounding areas. So far, numerous photothermal materials, such as noble metals represented by gold and silver, inorganic semiconductor materials, carbon materials and organic dyes, have been used in the research of near-infrared photothermal treatment of cancer. Among said materials, gold nanorods are the most widely used photothermal materials due to the good biocompatibility, adjustable spectrum and easy preparation thereof.

In the present study, a novel DDS for IDD was developed. The delivery subject contains three functional components: 1) DSPE-PEG ${ }_{2000}$-Folate (FA) was used to prepare folate response thermo-sensitive liposomes (FA-TMLs). FATMLs can deliver DOX to tumor tissue without causing toxicity to normal tissue, while FA can recognize the specific receptors on the surface of tumor cells; 2) magnetic nanoparticles (MNPs) can respond to the magnetic field applied to the target tumor tissue, thereby achieving effective enrichment of nano-drugs; and 3) gold nanorods (GNRs), which have a photothermal enhancing therapeutic effect through thermodynamic therapy. The three components were encapsulated in thermosensitive liposomes (TMLs) to form FATMLs@MNPs-GNRs-DOX (Scheme 1).FA-TMLs prevent the exposure of DOX to healthy cells in the process of delivery, then dissociate at the target tissue triggered by near-infrared irradiation to release DOX. To prepare the DDS, a microfluidic mixer chip is used. The DDS was characterized by DLS, TEM, UV-vis-NIR spectra and XRD. The drug release of the DDS was evaluated at room temperature and $45{ }^{\circ} \mathrm{C}$, which could be achieved by the 
photothermal effect through irradiation using a $980 \mathrm{~nm}$ laser beam (triggering structural changes of the FA-TMLs). The release of the drug achieved $95 \%$, and the results of cell uptake experiments reveal that FA-TMLs@MNPs-GNRsDOX could specifically bind folate receptor-positive cells and significant toxicity to bladder tumor cells with a cell viability of only $7 \%$.

\section{Materials and Methods}

\section{The Cell Culture}

The human bladder cancer cell line 5637 and lung cancer cell line A549 were obtained from China Center for Type Culture Collection. All cell culture media and supplements were obtained from Gibco. The human bladder cancer cell was grown in RPMI-1640. All media were supplemented with $10 \%$ fetal bovine serum (FBS) and 1\% penicillin -streptomycin. Cells were grown at $37{ }^{\circ} \mathrm{C}$ and $5 \% \mathrm{CO}_{2}$ (CLM-170B-8-NF, ESCO). Cells were harvested using accutase to ensure that surface antigens are not degraded by prolonged exposure to traditional reagents, such as trypsin-EDTA.

\section{Preparation of $\mathrm{Fe}_{3} \mathrm{O}_{4}$ Magnetic Nanoparticles}

MNPs were prepared through the chemical co-precipitation method. ${ }^{36,37}$ Briefly, $9.35 \mathrm{~g}$ of $\mathrm{FeCl}_{3} \cdot 6 \mathrm{H}_{2} \mathrm{O}$ and 3.44 $\mathrm{g}$ of $\mathrm{FeCl}_{2} \cdot 4 \mathrm{H}_{2} \mathrm{O}$ were dissolved in $200 \mathrm{~mL}$ of ultrapure water under nitrogen with vigorous stirring at $85{ }^{\circ} \mathrm{C}$. Subsequently, $40 \mathrm{~mL}$ of $30 \%$ (v/v) $\mathrm{NH}_{3} \cdot \mathrm{H}_{2} \mathrm{O}$ was added and the solution was heated for another $30 \mathrm{~min}$. After cooling, the MNPs were sequentially washed with ultrapure water and ethanol several times. The cleaned MNPs were stored in ethanol at a concentration of $40 \mathrm{~g} \mathrm{~L}^{-1}$.

\section{Preparation of Gold Nanorods}

GNRs were synthesized in accordance with a previously published procedure. ${ }^{38,39}$ First, CTAB-capped $\mathrm{Au}$ seeds were prepared by chemical reduction of $\mathrm{HAuCl}_{4}$ with $\mathrm{NaBH}_{4}$. $8.5 \mathrm{~mL}$ of $0.1 \mathrm{M} \mathrm{CTAB}$ was mixed with $94 \mu \mathrm{L}$ of $10 \mathrm{mM}$ $\mathrm{HAuCl}_{4}$, and the volume was fixed to $9.4 \mathrm{~mL}$ by adding water. Then, a $0.6 \mathrm{~mL}$ ice-cold $\mathrm{NaBH}_{4}$ aqueous solution $(0.01 \mathrm{M})$ was added to the aforementioned mixture. Seeds formed immediately and were used within $2-5 \mathrm{~h}$. The growth solution for GNRs consisted of a mixture of $10 \mathrm{~mL}$ of $0.1 \mathrm{M} \mathrm{CTAB}$, $208 \mu \mathrm{L}$ of $10 \mathrm{mM} \mathrm{HAuCl}_{4}, 90 \mu \mathrm{L}$ of $10 \mathrm{mM} \mathrm{AgNO}_{3}, 2$ drops of $\mathrm{HCl}$, and $800 \mu \mathrm{L}$ of $0.1 \mathrm{M}$ ascorbic acid. Growth was initiated by adding $240 \mu \mathrm{L}$ of seeds, and the temperature of the growth medium was kept constant at $26^{\circ} \mathrm{C}$ during the full procedure. The prepared GNRs had a longitudinal surface plasma resonance band centered at $980 \mathrm{~nm}$.

\section{Synthesis of DSPE-PEG 2000 -Folate (DSPE-PEG 2000 -FA)}

Folate-modified DSPE-PEG 2000 molecular was synthesized through a previously reported method with minor modifications. $^{40}$ The synthetic route (Figure S1) is presented in the supporting information. A solution of folate (220 mg, $0.5 \mathrm{mmol})$ in DMSO $(6 \mathrm{~mL})$ was stirred at $25^{\circ} \mathrm{C}$ for $1 \mathrm{~h}$. NHS (56.3 mg, $0.5 \mathrm{mmol})$, DCC (200 mg, 1 mmol), and triethylamine (TEA $80 \mu \mathrm{L}$ ) were then added successively. The resulting precipitate (Folate-NHS) was collected and washed with cold acetone and anhydrous diethyl ether three times. Subsequently, a mixture of Folate-NHS (69 mg, $0.125 \mathrm{mmol}$ ), DSPE-PEG $2000-\mathrm{NH}_{2}$ (343 mg, $0.125 \mathrm{mmol}$ ), DMSO (4 mL) and pyridine (400 $\mu \mathrm{L}$ ) was reacted at room temperature (wrapped with $\mathrm{Al}$ foil) with nitrogen protection for $12 \mathrm{~h}$. After removal of the pyridine by vacuum distillation, the sample was dissolved in water $(10 \mathrm{~mL})$ and centrifuged to remove insoluble particulate. Finally, DSPE-PEG 2000 -Folate was obtained by dialysis purification. The target molecule was characterized by Mass Spectroscopy (Waters Xevo G2-Q-Tof) (Figure S2).

\section{Preparation of Thermosensitive Liposomes by Microfluidics}

Thermosensitive liposomes (TMLs) were prepared by a microfluidic mixer chip and used to encapsulate MNPs, GNRs and DOX. Dipalmitoylphosphatidylcholine (DPPC), cholesterol, 1,2-distearoyl-sn-glycero-3-phosphoethanolamine-N-[amino(polyethylene glycol)-2000] (DSPE-PEG 2000 ), DSPE-PEG $2000-\mathrm{FA}$ (mol ratio= 90:5:3.5:1.5) and MNPs were mixed with methanol as Solution A (methanol phase). DOX and GNRs were dispersed in the PBS solution as Solution B (Aqueous phase). Then, the Solution A and Solution B were injected simultaneously into the micromixer using a syringe pump. All formulations were prepared at $4 \mathrm{mg} / \mathrm{mL}$ initial lipid concentration at 4:1 (aqueous: organic) flow rate ratio (FRR) and $25 \mathrm{~mL} / \mathrm{min}$ total flow rate (TFR). All newly formed liposomes $(8 \mathrm{~mL})$ were then subjected to buffer exchange via dialysis against $1 \times \mathrm{PBS} \mathrm{pH} 7.4$ for $1 \mathrm{~h}$ under magnetic stirring. 


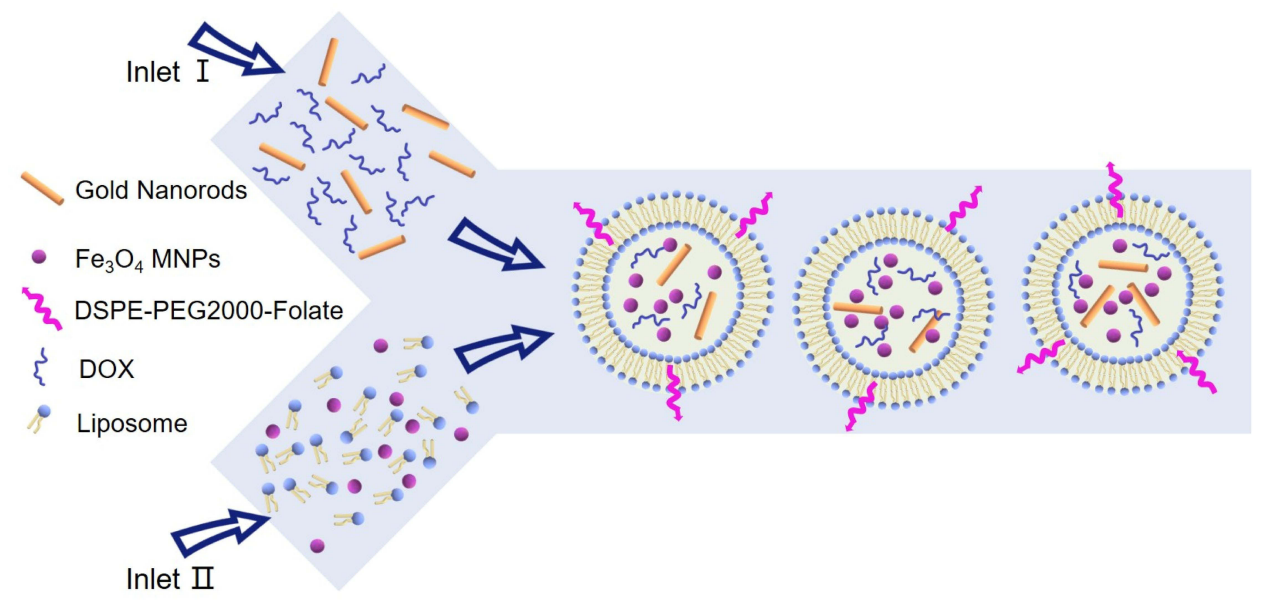

Scheme I A schematic illustration of the one-step microfluidic fabrication of magnetic and photothermal-responsive liposomes (FA-TMLs@MNPs-GNRs-DOX).

\section{Characterization of Thermosensitive Liposomes}

The size, polydispersity (PDI) and zeta potential of liposomes were determined by dynamic light scattering (DLS) using a Zetasizer Nano-ZS (Malvern Instruments Ltd). The morphology and particle size of MNPs, GNRs and liposomes were determined by transmission electron microscope (TEM, JEM-2100F). Differential scanning calorimetry (DSC) was used to study the thermal behavior of liposomes. A $980 \mathrm{~nm}$ continuous wave (CW) laser (Beijing Hi-Tech Optoelectronic Co., Ltd.) was used as the excitation source, with a thermal electric coupling thermometer (Eppendorf) to record the temperature changes. The MTT test was performed on a microplate reader (Spectrophotometer Varioskan LUX). Magnetic hysteresis loops were measured with a vibrating sample magnetometer (MPMS XL-7, Quantum Design).

\section{Encapsulation Efficiency and Photothermal-Responsive Drug Release}

By using a UV spectrophotometer at $460 \mathrm{~nm}$, the encapsulation efficiency (EE) of DOX in the sample was determined. The EE (\%) was calculated by the following equation: $\mathrm{EE}(\%)=\mathrm{W}_{\mathrm{E}} \mathrm{W}_{\mathrm{T}} \times 100 \%$, where $\mathrm{W}_{\mathrm{E}}$ represents the content of DOX encapsulated in the liposomes, and $\mathrm{W}_{\mathrm{T}}$ represents the content of total DOX added in the preparation. The drug release of FA-TMLs@MNPsGNRs-DOX under different temperature conditions and NIR light irradiation was examined by using a dynamic dialysis method. ${ }^{41,42}$ Briefly, 1 mL FA-TMLs@MNPsGNRs-DOX solution was added into the dialysis bag, and immersed in a beaker containing PBS (at temperature of 25,37 and $50{ }^{\circ} \mathrm{C}$ ). The dialysate was sampled at different time points. For photothermal mediated release, the FA-TMLs@MNPs-GNRs-DOX solution was then subjected to NIR laser irradiation $(980 \mathrm{~nm})$ and ultraviolet absorption intensity was measured in a spectrometer intermittently at various time periods. For $100 \%$ release, FATMLs@MNPs-GNRs-DOX was treated with 1\% Triton $\mathrm{X}-100$. The percentage release of DOX was calculated by the following formula, Release $\%=\left[\left(A_{t}-A_{i}\right) /\left(A_{f}-A_{i}\right)\right]$ $\times 100 \%$, where $A_{t}=$ absorbance at time $t, A_{i}=$ initial absorbance, $A_{f}=$ absorbance with $100 \%$ release, ie Triton$\mathrm{X}-100$ treatment.

\section{Toxicity and Antitumor Effect in vitro of Materials}

The cytotoxicity and antitumor effect in vitro of FATMLs, FA-TMLs@MNPs-GNRs, and FA-TMLs@MNPsGNRs-DOX were measured using the MTT method with the human bladder cancer cell line 5637. Bladder cancer cells with a density of $1 \times 10^{4}$ cells/well were incubated with FA-TMLs, FA-TMLs@MNPs-GNRs and FATMLs@MNPs-GNRs-DOX (the volume ratio of liposome to cell culture medium was $1: 99$ ) for $24 \mathrm{~h}$ and then exposed to a $980 \mathrm{~nm}$ NIR laser of $0,0.3,0.5$ and $1 \mathrm{~W} /$ $\mathrm{cm}^{2}$ for $15 \mathrm{~min}$. Every duration of NIR light irradiation was $3 \mathrm{~min}$, and the NIR laser was turned off for $3 \mathrm{~min}$. To evaluate the toxicity of NIR light irradiation, cells incubated on the FA-TMLs@MNPs without GNRs were also exposed to NIR light. Then, $20 \mu \mathrm{L}$ of MTT $(5 \mathrm{mg} / \mathrm{mL})$ was added into the wells and further incubated for an additional $4 \mathrm{~h}$. Subsequently, the supernatant was discarded, 
followed by the addition of $100 \mu \mathrm{L}$ of DMSO into each well and incubation in the shaker incubator with gentle shaking. The optical density (OD) was then read at a wavelength of $490 \mathrm{~nm}$. Relative inhibition of cell growth was expressed as follows: Relative inhibition $\%=(1-$ [OD]test/[OD]control) $\times 100 \%$.

\section{Localization of DOX in Subcellular of Bladder Tumor Cells}

After FA-TMLs@MNPs-GNRs-DOX irradiation incubation, the subcellular distribution of DOX in bladder tumor cells was observed by laser confocal microscopy. The cells were incubated with LysoTracker green (50 $\mathrm{nmol} / \mathrm{L})$ for $1 \mathrm{~h}$ at $24{ }^{\circ} \mathrm{C}$. After washing, the cells were fixed with $4 \%$ paraformaldehyde (30 $\mathrm{min}$ ) and stained with $10 \mu \mathrm{g} / \mathrm{mL}$ DAPI (blue) for $30 \mathrm{~min}$ at $24{ }^{\circ} \mathrm{C}$. The cells were imaged by a Zeiss LSM 710 confocal microscope (Carl Zeiss Microscope systems, Germany) with identical settings for each confocal study.

\section{In vitro Hemolysis Study}

All the experimental protocols were approved by Changzhou University Ethics Committee following the principles of laboratory and animal care of the University (CCZUIACUC-AP-2020010473) and each participant gave written informed consent. We obey the principles of the 1983 Declaration of Helsinki. In other words, all of the experiments in this paper obey this principle. In the hemolysis assay, the healthy human whole blood was obtained from Renmin Hospital of Wujin. Human red blood cells (hRBCs) were collected by centrifugation ( $3000 \mathrm{rpm}, 5 \mathrm{~min}$ ) of the blood and diluted ten times with PBS. Test materials prepared in PBS were co-incubated with diluted hRBCs for $2 \mathrm{~h}$ at $37^{\circ} \mathrm{C}$ with gentle mixing and centrifuged at $3000 \mathrm{rpm}$ for $5 \mathrm{~min}$. Further, $100 \mu \mathrm{L}$ aliquots of the resulting supernatant were transferred into a 96-well plate and hemoglobin release was recorded by the absorbance at $540 \mathrm{~nm}$ with a microplate reader (Varioskan LUX). The fresh hBCs in PBS and in $0.1 \%$ Triton X-100 acted as the negative and positive controls, respectively. The hemolysis activity was calculated using the following equation: Hemolysis activity $\%=\left(\mathrm{Abs}_{540} \mathrm{~nm}\right.$ with lipidosome $-\mathrm{Abs}_{540 \mathrm{~nm}}$ in $\mathrm{PBS}) /\left(\mathrm{Abs}_{540} \mathrm{~nm}\right.$ with $0.1 \%$ Triton $\left.\mathrm{X}-100-\mathrm{Abs}_{540 \mathrm{~nm} \text { in } \mathrm{PBS}}\right) \times 100 \%$.

\section{Statistical Analysis}

Statistical analysis of the data was performed using SPSS 16.0 (SPSS Inc.). All results of at least three parallel samples were expressed as mean \pm standard deviation (SD). Statistical significance was present as $* * \mathrm{P}<0.01 ; * * * \mathrm{P}<0.001$.

\section{Results \\ Characteristics of $\mathrm{Fe}_{3} \mathrm{O}_{4}$ Magnetic Nanoparticles, Gold Nanorods and DSPE-PEG 2000 -FA}

The chemical co-precipitation method was used to create MNPs known to be stable and biocompatible. The results of TEM image (Figure 1A) and DLS analyses (Figure 1B) reveal that the MNPs were $10 \mathrm{~nm}$ in diameter. Through comparison with the data from JCPDS file (01-076-1849), the powder X-ray diffraction (XRD) pattern in Figure 1C shows that the as-synthesized MNPs had a face-centered cubic (fcc) phase, indicating the formation of $\mathrm{Fe}_{3} \mathrm{O}_{4}$ crystals. The peaks at $2 \theta=30.2^{\circ}, 35.5^{\circ}, 43.2^{\circ}, 53.5^{\circ}, 56.8^{\circ}$, and $62.7^{\circ}$ could be indexed to (220), (311), (400), (422), (511), and (440) lattice planes of cubic magnetite, respectively. ${ }^{43}$ No impurity peaks were observed, indicating a pure phase of the sample. The IR spectrum for the MNPs exhibited a stretching vibration at $3420 \mathrm{~cm}^{-1}$, incorporating the contributions from both symmetrical and asymmetrical modes of the $\mathrm{O}-\mathrm{H}$ bonds which were attached to the surface iron atoms (Figure S3) ${ }^{44}$ The presence of MNPs was revealed in IR by the strong absorption band at around $587 \mathrm{~cm}^{-1}$, which could be attributed to the $\mathrm{Fe}-\mathrm{O}$ bond of MNPs. ${ }^{44}$ Additionally, the magnetic hysteresis loop analysis and time-dependent magnetic separation efficiency are shown in Figure S4 and S5. The MNPs had a high magnetization (49 emu/g), which enhanced the response thereof to the external magnetic field, and thus, should greatly facilitate the manipulation of the drugs in practical use. ${ }^{45}$

GNRs were synthesized according to the gold seed

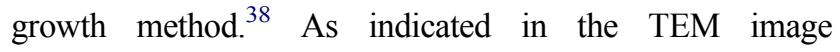
(Figure 1D), the GNRs exhibited a rod morphology with dimensions of $\sim 41 \mathrm{~nm} \times 7 \mathrm{~nm}$ (aspect ratio of $\sim 5.9$ ). The UV-vis-NIR spectra demonstrate that GNRs had an apparent surface plasmonic resonance band at $\sim 980 \mathrm{~nm}$ in the NIR region (Figure 1E), being conductive to photothermal transfer. The corresponding XRD pattern of the GNRs is shown in Figure $\mathrm{S}^{38} .{ }^{38}$ The temperature changes of the aqueous suspension of GNRs (Figure 1F) at different concentrations and time points measured, so as to investigate the temperature response of the GNRs to the irradiation of a diode laser at $980 \mathrm{~nm}$. The temperature of the control group exhibited a mild temperature change (only $\sim 2{ }^{\circ} \mathrm{C}$ ), while the temperature of the aqueous suspension of $150 \mu \mathrm{M}$ GNRs increased 

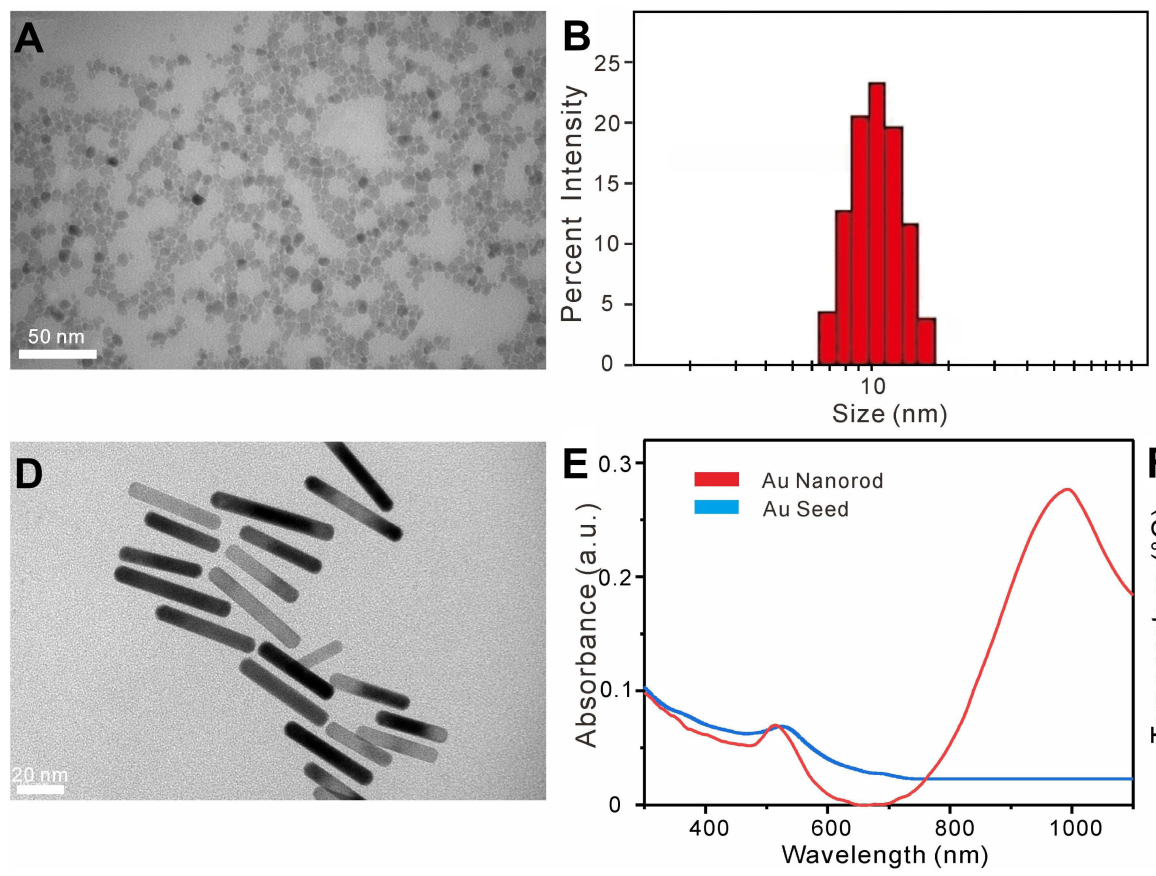
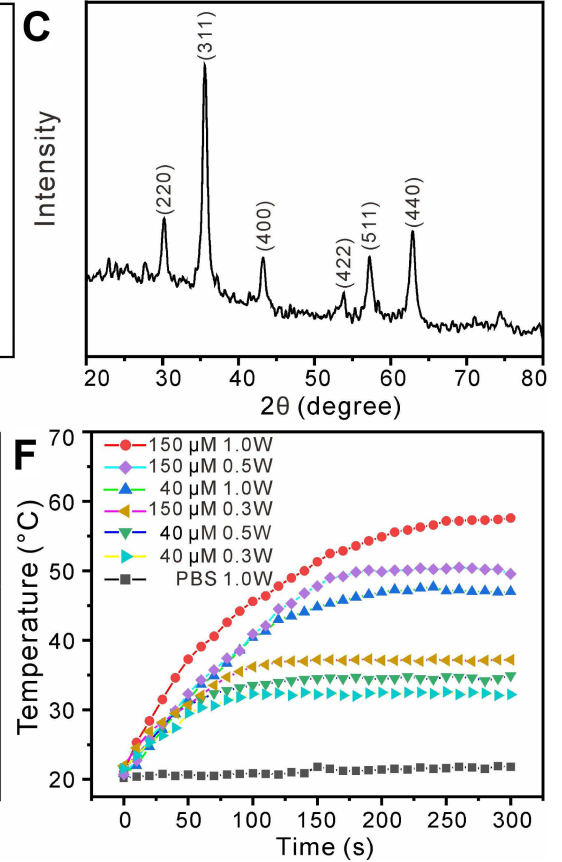

Figure I Preparation and characterization of $\mathrm{Fe}_{3} \mathrm{O}_{4}$ magnetic nanoparticles (MNPs) and gold nanorods $(\mathrm{GNRs}) .(\mathbf{A})$ TEM image of MNPs $($ scale bar $=50$ nm). $($ B) Particle size of MNPs. (C) XRD patterns of MNPs. (D) TEM image of GNRs (scale bar $=20 \mathrm{~nm}$ ). (E) UV-vis-NIR spectra of GNRs. (F) Temperature-responsive curves of a GNRs solution treated with different NIR irradiation.

with the irradiation time, reaching 46,50 , and $55^{\circ} \mathrm{C}$ in $5 \mathrm{~min}$ at irradiation powers of $0.3,0.5$, and $1.0 \mathrm{~W} / \mathrm{cm}^{2}$, respectively.

To obtain the DSPE-PEG 2000 -Folate, carbodiimide chemistry was used for amine cross-linking of DSPE$\mathrm{PEG}_{2000}-\mathrm{NH}_{2}$ with the carboxylic acid groups on the folate (Figure S1). As shown in Figure S2, the major multi-peaks centered at a 3195 mass-charge ratio indicate that the mean MW of DSPE-PEG 2000 -folate was consistent with the theoretical data (3195).

\section{Characteristics of the Liposomal Formulations}

FA-TMLs were prepared through a microfluidic mixer chip (Figure S7). Different flow rate ratios (FRR) and total flow rates (TFR) were investigated for liposome preparation, so as to optimize the preparation of FA-TMLs. The results shown in Figure $2 \mathrm{~A}$ and $\mathrm{B}$ demonstrate that with the increase of FRR and TFR, the particle size of liposomes became smaller, which could be attributed to the rapid mixing caused by high flow rates at high Reynolds numbers. The structure of FA-TMLs@MNPsGNRs-DOX characterized by TEM exhibited spherical morphology with $\sim 230 \mathrm{~nm}$ size, with few discrete MNPs and GNRs in the aqueous core and enclosed within a lipid bilayer (Figure 2C). The DSC calorimetric profiles reveal that the transition temperature of FA-TMLs was $44^{\circ} \mathrm{C}$ and the drug could be released above body temperature $\left(37^{\circ} \mathrm{C}\right)$ (Figure 2D). The sizes of FA-TMLs, FA-TMLs@MNPsGNRs, and FA-TMLs@MNPs-GNRs-DOX are shown in Figure S8 and Table S1). Moreover, the FATMLs@MNPs-GNRs-DOX were stable as the particle size and PDI did not significantly increase after 3 months of storage (Figure 2E).

The results of magnetic hysteresis loop analysis at room temperature indicate a saturation magnetization value of $49 \mathrm{emu} / \mathrm{g}$ for MNPs and $20 \mathrm{emu} / \mathrm{g}$ for FATMLs@MNPs-GNRs-DOX (Figure 2F). The encapsulation efficiency of DOX in FA-TMLs was affected by three factors: 1) the concentration of DOX; 2) the concentration of MNPs; and 3) the concentration of GNRs. MNPs and GNRs affect the formation and particle size of liposomes. As indicated in Figure $2 \mathrm{G}$, with an increase of the concentration of DOX, the encapsulating efficiency of DOX decreased from $79.0 \% \pm 5.5 \%$ to $28.2 \% \pm 6.3 \%$. When the concentration of MNPs was $0.05 \mathrm{mg} / \mathrm{mL}$, the maximum encapsulating efficiency of DOX was $43.3 \% \pm$ $5.9 \%$ and the loading efficiency was $29.8 \% \pm 3.3 \%$ (Figure 2H). Similarly, Figure S9 shows that the encapsulation of DOX in FA-TMLs could be obtained at $300 \mu \mathrm{M}$ GNRs, which simultaneously provided the maximal 
encapsulation efficiency $(28.6 \% \pm 4.6 \%)$ and loading efficiency $(22 \% \pm 6 \%)$. In conclusion, the conditions of $2 \mathrm{mg} /$ $\mathrm{mL}$ DOX, $0.05 \mathrm{mg} / \mathrm{mL}$ MNPs and $300 \mu \mathrm{M}$ GNRs were optimal for subsequent experiments.

\section{Drug Release in vitro and Cellular Uptake of FA-TMLs@MNPs-GNRs-DOX}

When the temperature of thermosensitive liposomes exceeds the phase transition temperature, the structure of FA-TMLs is transformed and destroyed to release drugs. ${ }^{46}$ The release of FA-TMLs@MNPs-GNRs-DOX was investigated through water bath heating and photothermal conversion experiments. As shown in Figure 3A, only about $16.3 \%$ of DOX was released from FA-TMLs@MNPs-GNRs-DOX after 30 min incubation at $24{ }^{\circ} \mathrm{C}$, whereas $52.2 \%$ of the drug was released after $24 \mathrm{~h}$. However, when the incubation temperature rose to $45^{\circ} \mathrm{C}, 90.1 \%$ of the drug was released after 30 min. Further drug release experiments were conducted in terms of photothermal transduction. Findings were made that after $980 \mathrm{~nm}$ light treatment, $85.4 \%$ of the drug was released after $30 \mathrm{~min}$ (Figure 3B). The methylthiazoletetrazolium (MTT) assay was used to test the effects of MNPs, GNRs and DOX on cell viability. As demonstrated in Figures $3 \mathrm{C}$ and $\mathrm{D}$, with increasing concentrations of FATMLs (0.1 to $2000 \mu \mathrm{g} / \mathrm{mL})$ and MNPs ( 0.01 to $0.2 \mathrm{mg} / \mathrm{mL})$, the bladder cancer cells did not lose viability or exhibit detectable toxicity after 24 hours of incubation, demonstrating that the synthesized liposomes have good biocompatibility. However, the results from DOX loaded liposomes reveal that cell viability decreased from $94.4 \%$ to $4.7 \%$ as the DOX concentration increased from $0.1 \mu \mathrm{M}$ to $7.5 \mu \mathrm{M}$ (Figure S10, S11).

The cell viabilities of FA-TMLs and FA-TMLs +980 $\mathrm{nm}$ hv were found to be $98.8 \pm 2.7 \%$ and $95.4 \pm 2.4 \%$ respectively, indicating that the irradiation had no obvious effect on the cells. The cell viabilities of FATMLs@MNPs-GNRs and FA-TMLs@MNPs-GNRs+ 980 nm hv were $97.6 \pm 3.5 \%$ and $74.4 \pm 4.6 \%$, respectively. In the experiment with FA-TMLs@MNPs-GNRs-DOX and FA-TMLs@MNPs-GNRs-DOX + 980 nm hv, the cell viabilities decreased from $29.7 \pm 3.5 \%$ of FATMLs@MNPs-GNRs-DOX to $7.4 \pm 3.6 \%$ of FATMLs@MNPs-GNRs-DOX + 980 nm hv (Figure 3E). At the same time, calcein AM and propidium iodide (PI) were used to stain the bladder cancer cells to analyze the viability. The assay-stained live cells with a green fluorescence and dead cells with a red fluorescence (Figure 4).

To further explore the targeting effect of folic acid, the cytotoxicity of the control group, TMLs@MNPs-GNRsDOX, FA-TMLs@MNPs-GNRs-DOX, and DOX were investigated to folate-negative A549 cells and folate-positive bladder cancer cells by MTT (Figure 3F). In A549 cells, the cell viabilities of TMLs@MNPs-GNRs-DOX, FA-TMLs@MNPs-GNRs-DOX and DOX were $61.45 \pm$ $5.23 \%, 58 \pm 4.75 \%$ and $35 \pm 4.3 \%$, respectively. In contrast, in bladder cancer cells 5637, the cell viability of TMLs@MNPs-GNRs-DOX, FA-TMLs@MNPs-GNRsDOX and DOX were $57.3 \pm 6.34 \%, 29 \pm 3.45 \%$ and 37

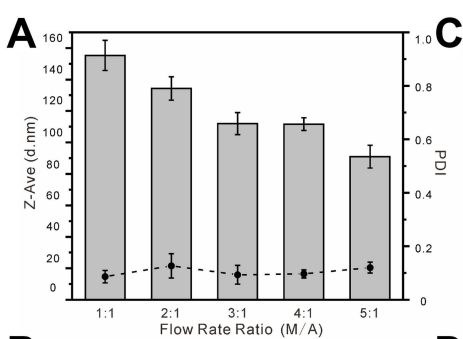

B

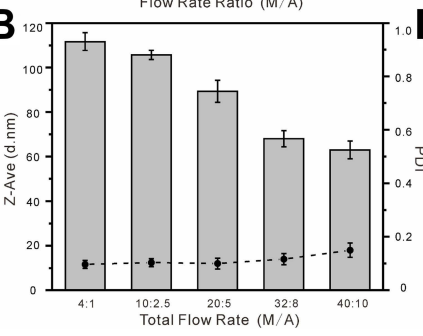

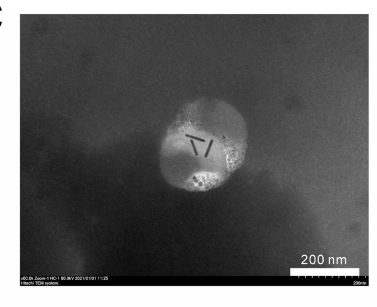

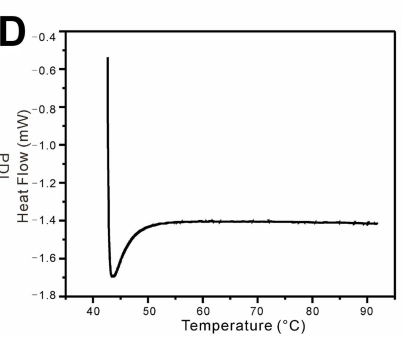

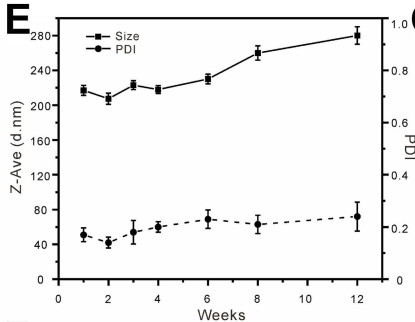

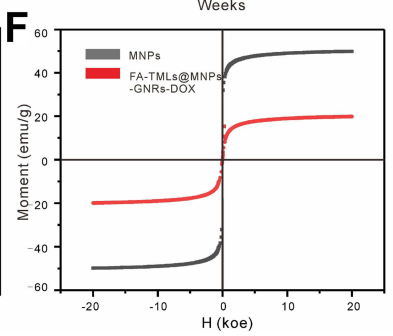

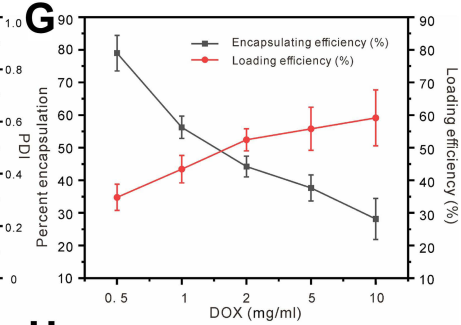

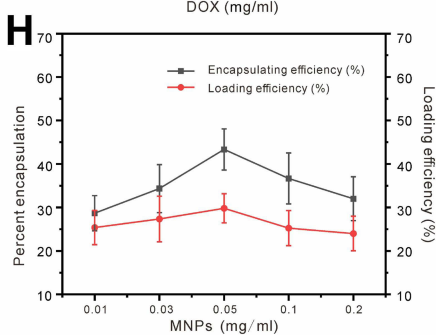

Figure 2 Preparation and characterization of folate-modified thermosensitive liposomes (FA-TMLs). (A) Effect of different flow rate ratio (MeOH solution: aqueous solution) on liposome size and PDI. (B) Effect of different total flow rate (MeOH solution: aqueous solution) on liposome size and PDI. (C) TEM image of FA-TMLs@MNPsGNRs. (D) DSC curve of FA-TMLs. (E) Stability of FA-TMLs@MNPs-GNRs-DOX (4 $\left.{ }^{\circ} \mathrm{C}\right)$. (F) Magnetic hysteresis loop of the MNPs and FA-TMLs@MNPs-GNRs-DOX. (G) The effects of DOX concentration on encapsulation efficiency and loading efficiency of DOX in FA-TMLs@MNPs-GNRs-DOX. (H) The effects of MNPs concentration on encapsulation efficiency and loading efficiency of DOX in FA-TMLs@MNPs-GNRs-DOX. 
$\pm 3.8 \%$, respectively. The cytotoxicity of FATMLs@MNPs-GNRs against normal mammalian cells was evaluated by the hemolytic assay using hRBCs from healthy volunteers, so as to evaluate the value of FATMLs@MNPs-GNRs in actual medication. ${ }^{47}$ Figure S12 showed the dose-response curves for the hemolytic activities of FA-TMLs@MNPs-GNRs. FA-TMLs@MNPsGNRs did not cause any hemolysis even at concentrations up to $2000 \mu \mathrm{g} / \mathrm{mL}$.

\section{Localization of DOX in Subcellular of Bladder Tumor Cells}

The images of bladder cancer cells treated with FATMLs@MNPs-GNRs-DOX under 980 nm irradiation are shown in Figure 5. After the drug entered the cells, the red fluorescence of DOX increased with the increase of incubation time, indicating gradual transfer of DOX from the lysosome to the nucleus. Thus, FA-TMLs@MNPs-GNRsDOX selectively entered the tumor cells with high expression of folate receptor through folate receptormediated endocytosis, and the DOX subsequently entered the cell nucleus and exerted an antitumor effect.

\section{Discussion}

Bladder cancer is the most common malignant tumor in the urinary system and is a serious threat to human health. At present, the main treatment is bladder infusion with chemotherapy drugs such as mitomycin, doxorubicin, hydroxycamptothecin, and others. However, despite the capability thereof of killing cancer cells, said drugs can also cause damage to normal tissues. In recent years, several targeted drug delivery systems have been developed to increase drug concentration at the focal site and reduce drug damage to normal tissues. Liposomes are hollow spheres made of lecithin and ceramide, with a bilayer structure like cell membrane. Due to the good biocompatibility and ability to deliver drugs to target sites, liposomes are the most widely used carrier materials in
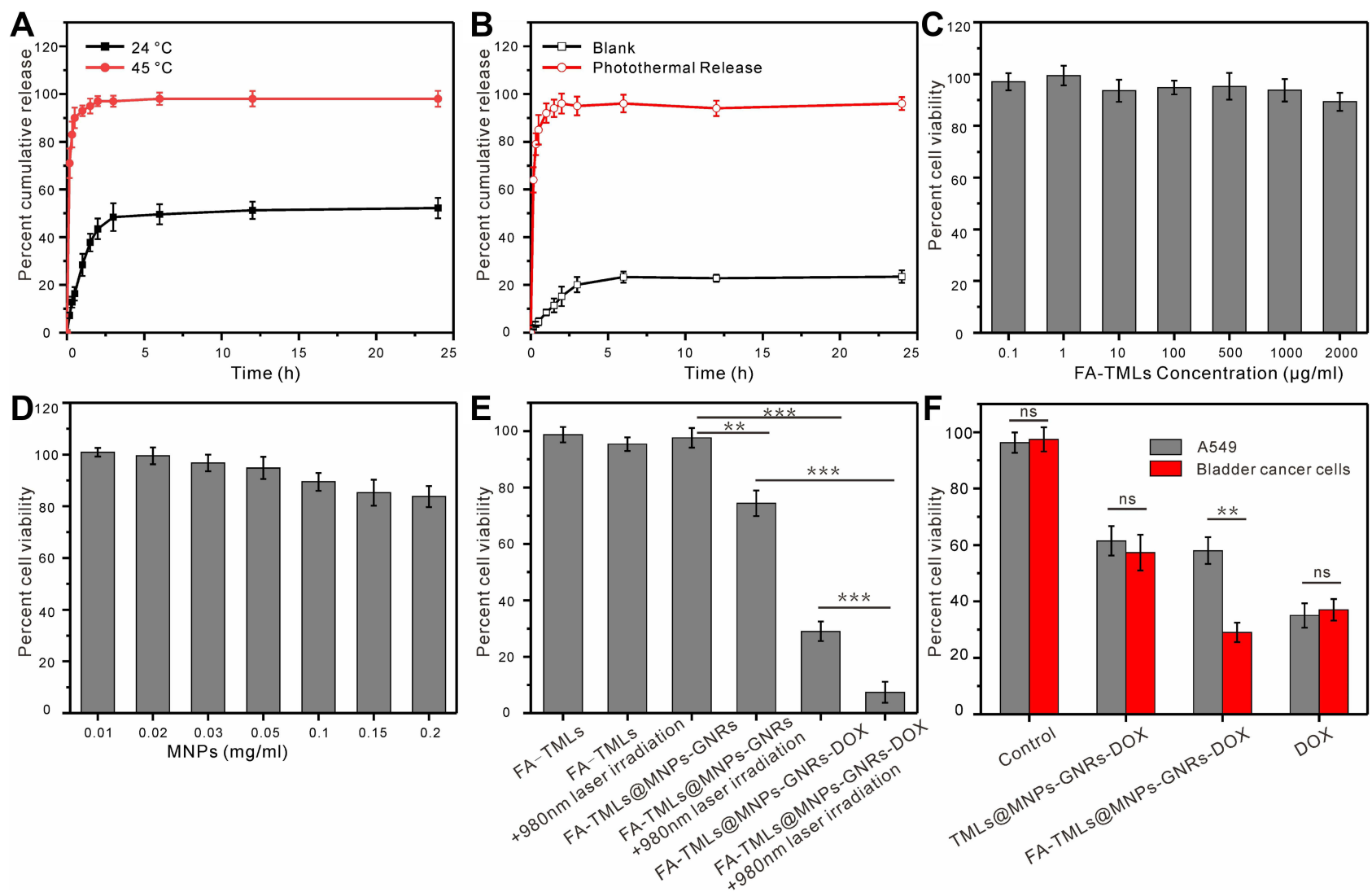

Figure 3 In vitro drug release and cellular uptake of FA-TMLs@MNPs-GNRs-DOX. (A) Temperature controlled DOX release from FA-TMLs@MNPs-GNRs-DOX. (Black: $24{ }^{\circ} \mathrm{C}$; Red: $45{ }^{\circ} \mathrm{C}$ ). (B) Photo-thermal controlled DOX release from FA-TMLs@MNPs-GNRs-DOX. (C and D) Cytotoxicity of FA-TMLs (C) and MNPs (D). (E) Cell viability with FA-TMLs; FA-TMLs + 980 nm laser irradiation; FA-TMLs@MNPs-GNRs; FA-TMLs@MNPs-GNRs + 980 nm laser irradiation; FA-TMLs@MNPs-GNRs-DOX; FA-TMLs@MNPs-GNRs-DOX + 980 nm laser irradiation. (F) Cytotoxicity of control group, TMLs@MNPs-GNRs-DOX, FA-TMLs@MNPs-GNRs-DOX, DOX, to folatenegative A549 cells (gray) and folate-positive bladder cancer cells (red). Significant differences are represented as $* *$ p $<0.0 \mathrm{I}$, ***p $<0.00 \mathrm{I}$; no significant difference is represented as ns. 


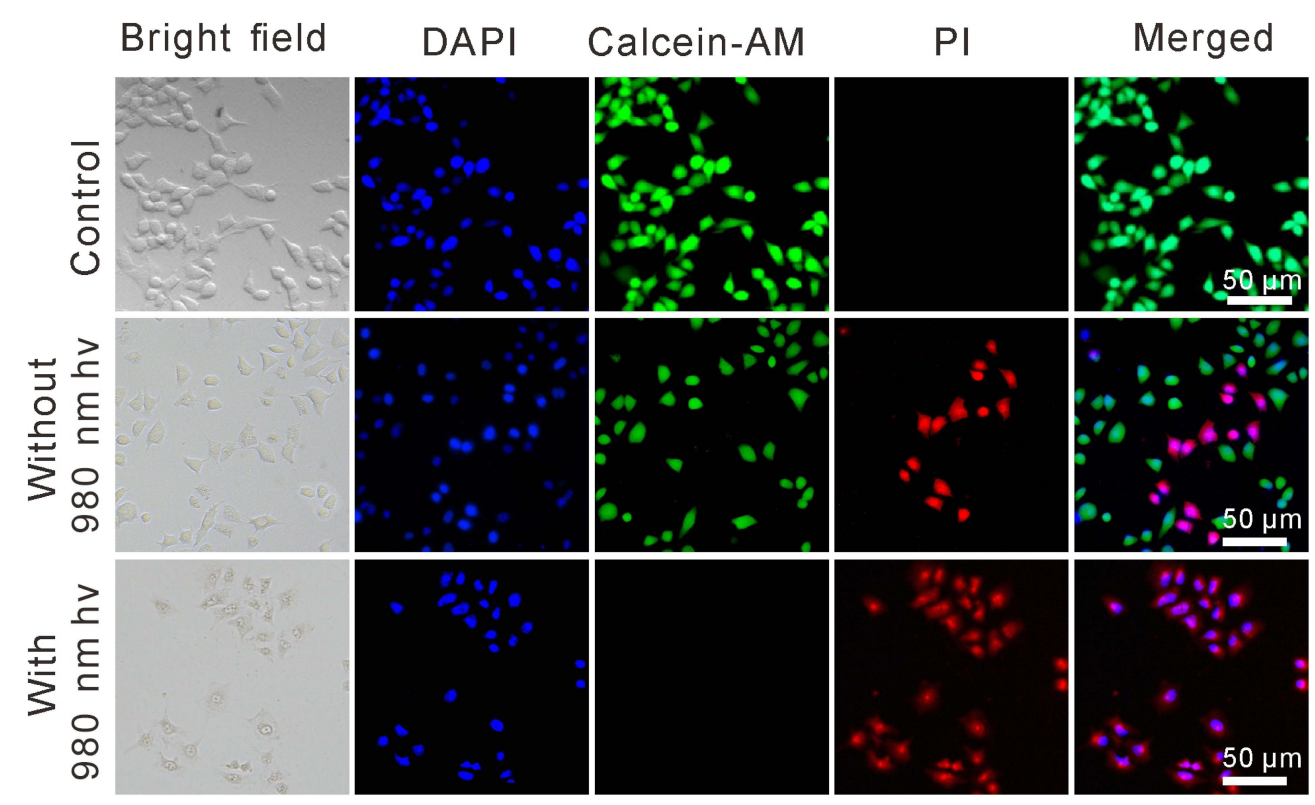

Figure 4 Fluorescence images of cells stained with calcein AM (green) and PI (red) incubated with the FA-TMLs@MNPs-GNRs-DOX without and with 980 nm NIR laser irradiation.

clinic. $^{48-50}$ In 1995, liposomal coated doxorubicin for the treatment of breast cancer was approved by the US Food and Drug Administration (FDA) as the first nanoagent (Doxil). ${ }^{51}$ In the present study, using a microfluidic hybrid chip, MNPs, GNRs and DOX were encapsulated in folic acid-modified thermosensitive liposomes to construct a multi-responsive liposome for targeted delivery of doxorubicin to bladder cancer cells. In further in vivo experiments, magnetic nanoparticles MNPs were able to enrich drug carriers in the lesion area by manipulating an external magnetic field. ${ }^{52}$ FA-TMLs can further deliver DOX to tumor cells without damage to normal tissue by recognizing tumor cell surface-specific receptors. GNRs can not only realize the release of DOX wrapped by photocontrolled liposome structure changes but also enhance the therapeutic effect through thermodynamic therapy and chemotherapy, owing to the significant photothermal effect thereof.

In the present study, MNPs were prepared through the chemical co-precipitation method. Characterization data

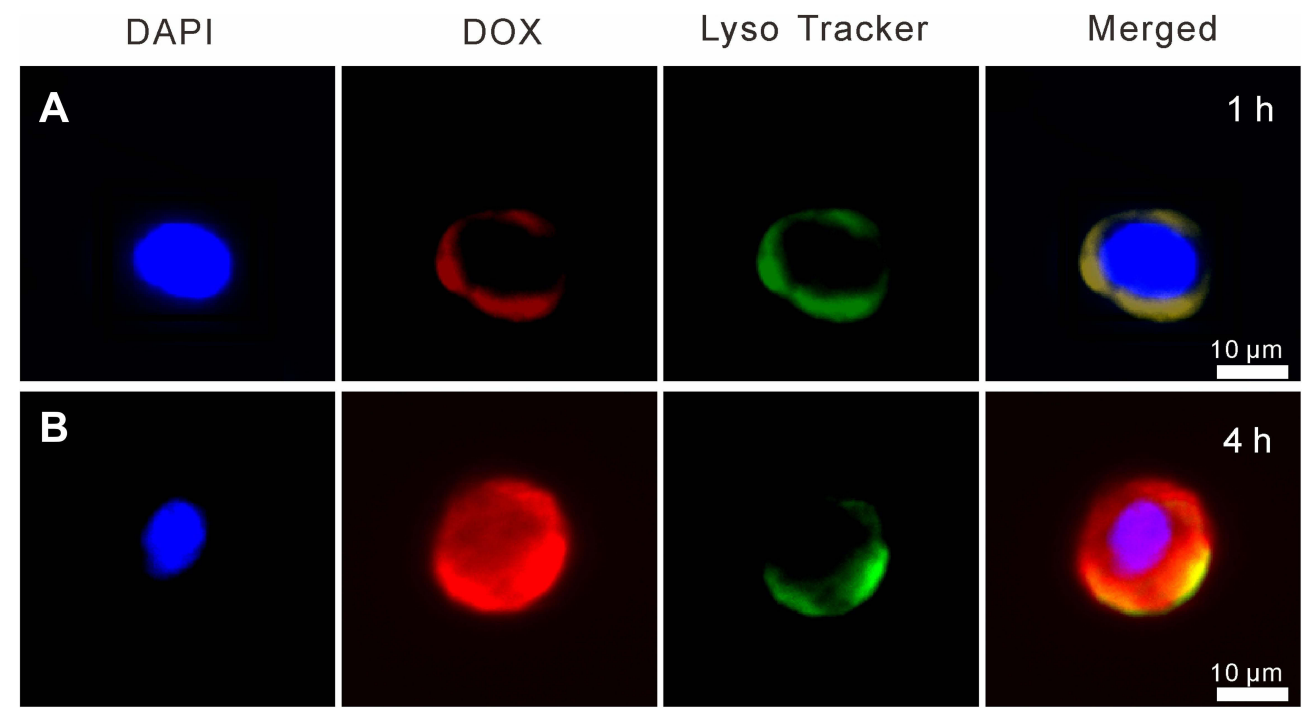

Figure 5 Localization of DOX in subcellular organelles of bladder tumor cells. The triple-labelling with the nucleus-selective dye (DAPI, blue), the fluorescent drug (DOX, red), and dyes selective for acidic endolysosomes (LysoTracker, green). (A) Ih; (B) $4 \mathrm{~h}$. 
reveal that the synthesized MNPs had a particle size of about 10nm and a face-centered cubic (fcc) phase. Additionally, the hysteresis loop analysis indicates that MNPs had a high magnetization (49 emu/g), and FATMLs@MNPs formed after liposome encapsulation had a magnetization of $20 \mathrm{emu} / \mathrm{g}$. Such findings could be attributed to the saturated magnetization diminishing in FA-TMLs@MNPs-GNRs-DOX due to the diamagnetic contribution of the lipid bilayer surrounding the magnetite, in addition to the low weight percentage of MNPs in FATMLs@MNPs-GNRs-DOX. The superparamagnetic behavior observed from the magnetization curve, wherein both the residual and coercivity forces were close to zero, allows for the possibility of using FA-TMLs@MNPsGNRs-DOX for magnetically targeted drug delivery.

Compared with photothermal materials such as semiconductors and organic dyes, gold nanomaterials have the advantages of good biocompatibility and low toxicity. Near-infrared light source is generally used in photothermal treatment of cancer to improve tissue penetration depth and reduce irradiation toxicity. Gold nanorods, ${ }^{38}$ gold nanoshell, ${ }^{53}$ gold nanocages, ${ }^{54}$ gold nanocube, ${ }^{55}$ gold nanostars ${ }^{56}$ and gold nanosphere oligomers ${ }^{57}$ with near-infrared photothermal response have been developed. In terms of the near-infrared light response, gold nanoshell, gold nanorods and gold nanocages have the strongest performance. The overall light extinction intensity of gold nanorods and gold nanocages is more than twice that of nanoshell, while the light absorption intensity of gold nanorods is slightly stronger than that of gold nanocages. Considering the difficulty of preparation, gold nanoshell and gold nanocages contain two kinds of components, require many steps and can take a long time. The preparation of gold nanorods can be completed in only two steps and can be mass-produced or even commercialized. Hence, gold nanorods with significant photothermal properties and biocompatibility were used in the present investigation. GNRs were synthesized according to the gold seed growing method. By controlling the concentration of silver nitrate, the aspect ratio of gold nanorods could be controlled, such that the GNRs had an obvious surface plasmon resonance band (about $980 \mathrm{~nm}$ ) in the near-infrared region. The temperature curves of GNRs aqueous solutions under $980 \mathrm{~nm}$ light irradiation at different concentrations and time points were investigated. The results suggest that the temperature of a FATMLs@GNRs could be easily controlled by adjusting the concentration of GNRs or NIR irradiation parameters (intensity or time). When controlling the conditions for which the irradiation power was $0.5 \mathrm{~W} / \mathrm{cm}^{2}$ and GNRs concentration was $150 \mu \mathrm{M}$, the temperature was kept at about $50{ }^{\circ} \mathrm{C}$. Thus, the following drug release experiments were conducted using such conditions. The folate receptors are over-expressed on prostate, lung, and colon cancer cells and have considerably low expression on normal cells. ${ }^{27,58}$ Such selective over-expression makes folate receptors notable tumor targets when conjugated to TMLs. In order to obtain FA-TMLs, DSPE-PEG 2000 -FA was synthesized by cross-linking DSPE-PEG $2000-\mathrm{NH}_{2}$ amine with carboxylic acid groups on folic acid using carbodiimide chemistry.

The FA-TMLs were prepared through a microfluidic mixer chip and used to encapsulate MNPs, GNRs and DOX. By investigating the influence of two significant parameters of flow rate ratio and total flow rate on the particle size and dispersion of the formed liposomes, the experimental conditions were set as follows: flow rate ratio of $4: 1$ and total flow rate of $25 \mathrm{~mL} / \mathrm{min}$. The structure of FA-TMLs@MNPs-GNRs-DOX characterized by TEM showed spherical morphology with $\sim 230 \mathrm{~nm}$ size, with few discrete MNPs and GNRs in the aqueous core and enclosed within a lipid bilayer. In the preparation of liposomes, microfluidic technology can replace the lipid hydration and extrusion steps with a single-step production process in which particle size is process-controlled. ${ }^{59-61}$ Further, the encapsulation and loading efficiency of DOX in liposomes can be adjusted by changing the concentration of drug and encapsulated nanoparticles. The encapsulation efficiency decreased with the increasing concentration of drug and encapsulated nanoparticles, while the loading efficiency was positively correlated with the drug concentration.

By conditional optimization, the conditions of $2 \mathrm{mg} /$ $\mathrm{mL}$ DOX, $0.05 \mathrm{mg} / \mathrm{mL}$ MNPs and $300 \mu \mathrm{M}$ GNRs were optimal for subsequent experiments. The concentration of DOX in the prepared FA-TMLs@MNPs-GNRs-DOX was $0.57 \mathrm{mg} / \mathrm{mL}$. The DSC calorimetric profiles reveal that the transition temperature of FA-TMLs was $44{ }^{\circ} \mathrm{C}$ and the drug could be released above body temperature $\left(37^{\circ} \mathrm{C}\right)$. Owing to the significant NIR photothermal effect of embedded GNRs, the selective release of drugs could be achieved through appropriate near-infrared (NIR) light irradiation. ${ }^{62}$ In order to verify such this conjecture, the drug release efficiencies of FA-TMLs@MNPs-GNRsDOX at NIR irradiation, $24{ }^{\circ} \mathrm{C}$ and $45^{\circ} \mathrm{C}$ were compared. The results reveal that the structure of FA-TMLs can be 
changed by $45^{\circ} \mathrm{C}$ water bath and light treatment, ultimately leading to rapid release of encapsulated drugs. The DOX release efficiency of $45{ }^{\circ} \mathrm{C}$ water bath and $980 \mathrm{~nm}$ light irradiation treatment for 30 minutes was $90.1 \%$ and $85.4 \%$, respectively. Such controlled and highly efficient nanocarrier could help deliver the drug adequately to the focal area, avoiding damage to normal tissue.

The effects of MNPs, GNRs and DOX on cell viability were first explored before the killing effect of FATMLs@MNPs-GNRs-DOX on bladder cancer cells was investigated. FA-TMLs $(0.1-2000 \mu \mathrm{g} / \mathrm{mL})$ and MNPs $(0.01-0.2 \mathrm{mg} / \mathrm{mL})$ exhibited no significant toxicity after being cultured with bladder cancer cells for $24 \mathrm{~h}$, respectively, indicating that the synthesized liposomes and MNPs had good biocompatibility. However, the MTT results of DOX reveal that the cell viability decreased from $94.4 \%$ to $4.7 \%$ when the DOX concentration was increased from 0.1 $\mu \mathrm{M}$ to $7.5 \mu \mathrm{M}$. To further study the cytotoxicity of the photothermal effect, the cell viabilities of FA-TMLs, FATMLs + 980 nm hv, FA-TMLs@MNPs-GNRs, FATMLs@MNPs-GNRs+ 980 nm hv, FA-TMLs@MNPsGNRs-DOX and FA-TMLs@MNPs-GNRs-DOX + 980 $\mathrm{nm}$ hv were evaluated. The volume ratio of FATMLs@MNPs-GNRs-DOX to cell culture medium was $1: 4$, and the final concentration of DOX was about 114 $\mu \mathrm{g} / \mathrm{mL}$. The results reveal that irradiation had no significant effect on cell viability. However, in the presence of GNRs, cell viability decreased from $97.6 \pm 3.5 \%$ to $74.4 \pm 4.6 \%$ under light irradiation, showing that irradiation with GNRs affected cell viability, and had a photothermal effect. In the experiments with FA-TMLs@MNPs-GNRs-DOX and FATMLs@MNPs-GNRs-DOX + 980 nm hv, the cell viabilities decreased from $29.7 \pm 3.5 \%$ of FA-TMLs@MNPs-GNRsDOX to $7.4 \pm 3.6 \%$ of FA-TMLs@MNPs-GNRs-DOX + $980 \mathrm{~nm}$ hv, revealing a typical enhanced DOX cytotoxicity with irradiation due to increased intracellular drug concentration after the photothermal triggered release of DOX from FA-TMLs@MNPs-GNRs-DOX. Meanwhile, calcein AM and PI were used to stain the bladder cancer cells to analyze the viability. Calcein AM can penetrate the live cell membrane and react with the intracellular esterase to form calcein with green fluorescence, while PI is a membrane-impermeable nuclear stain that can stain only dead cells, resulting in red fluorescence. ${ }^{63}$ An observation can be made that more than half of the bladder cancer cells with FATMLs@MNPs-GNRs-DOX exhibited green fluorescence, while the cells with FA-TMLs@MNPs-GNRs-DOX + 980 $\mathrm{nm}$ hv only exhibited red fluorescence.

To further study the targeting effect of folic acid, the cytotoxicity of the control group, TMLs@MNPs-GNRsDOX, FA-TMLs@MNPs-GNRs-DOX, and DOX were investigated to folate-negative A549 cells and folate-positive bladder cancer cells 5637 by MTT. In A549 cells, TMLs@MNPs-GNRs-DOX, FA-TMLs@MNPs-GNRsDOX and DOX exhibited similar cytotoxicity to the two cell lines. In contrast, FA-TMLs@MNPs-GNRs-DOX exhibited greater cytotoxicity to bladder cancer cells 5637 than TMLs@MNPs-GNRs-DOX. Such results indicate that FA-TMLs@MNPs-GNRs-DOX could selectively act on tumor cells with high expression of folate receptor and enhance the antitumor effect of the drug. ${ }^{64}$ In order to further study the drug toxicity of nanocarriers, the cytotoxic activity of DOX, TMLs@MNPs-GNRs-DOX, FATMLs@MNPs-GNRs-DOX and FA-TMLs@MNPsGNRs-DOX + irradiation against bladder cancer 5637 cells and A549 cells was evaluated (Table 1). In 5637 cells, FA-TMLs@MNPs-GNRs-DOX + irradiation was the most effective treatment $\left(\mathrm{IC}_{50}, 2.8 \mu \mathrm{M}\right)$. The cytotoxicity of said preparation was twice that of TMLs@MNPs-GNRsDOX and even more than that of free DOX. On the other hand, the A549 cells did not express folate receptors, and TMLs@MNPs-GNRs-DOX and FA-TMLs@MNPs-GNRsDOX exhibited similar cytotoxicity. The photothermal effect could increase the toxicity of FA-TMLs@MNPsGNRs-DOX in 5637 cells and A549 cells. In order to evaluate the value of FA-TMLs@MNPs-GNRs in actual medication, the cytotoxicity of FA-TMLs@MNPs-GNRs

Table I Cytotoxicity for Bladder Cancer 5637 Cells and A549 Cells Treated with DOX, FA-TMLs@MNPs-GNRs-DOX, FATMLs@MNPs-GNRs-DOX + Irradiation

\begin{tabular}{|l|c|c|}
\hline Drugs & IC $_{\mathbf{5 0}}(\boldsymbol{\mu} \mathbf{M}) / \mathbf{5 6 3 7}$ Cells & IC $_{\mathbf{5 0}}(\boldsymbol{\mu} \mathbf{M}) / \mathbf{A 5 4 9}$ Cells \\
\hline DOX & $3.2 \pm 0.4$ & $3.0 \pm 0.5$ \\
TMLs@MNPs-GNRs-DOX & $6.3 \pm 1.2$ & $6.0 \pm 1.4$ \\
FA-TMLs@MNPs-GNRs-DOX & $4.4 \pm 0.8$ & $5.9 \pm 1.1$ \\
FA-TMLs@MNPs-GNRs-DOX + irradiation & $2.8 \pm 0.6$ & $4.2 \pm 0.9$ \\
\hline
\end{tabular}


against normal mammalian cells was evaluated by the hemolytic assay using hRBCs from healthy volunteers. ${ }^{47}$ The hemolysis curve reveals that FA-TMLs@MNPs-GNRs did not cause hemolysis even when the concentration reached $2000 \mu \mathrm{g} / \mathrm{mL}$. Such results indicate that FATMLs@MNPs-GNRs-DOX had potential applications in vivo.

To demonstrate the distribution of DOX in the nucleus and endosome/lysosome, cells were stained with DAPI (blue) and LysoTracker (green). ${ }^{65,66}$ An observation can be made that the drugs were mainly distributed in lysosomes after entering cells through endocytosis at the early stage of drug uptake $(1 \mathrm{~h})$. With the increasing incubation time (4 h), DOX was gradually transferred from lysosome to nucleus. As such, FA-TMLs@MNPs-GNRs-DOX selectively entered the bladder cancer cells 5637 with high expression of folate receptor through folate receptor-mediated endocytosis, and the DOX subsequently entered the cell nucleus and exerted an antitumor effect.

\section{Conclusion}

In conclusion, magnetically responsive and folate-receptor targeted TMLs for antitumor drug (DOX) delivery synergically with a photothermal effect were successfully developed for the treatment of bladder cancer. TMLs with uniform particle size were prepared through a microfluidic chip. The morphology and properties of the multi-functionalized FA-TMLs@MNPs-GNRs-DOX were verified by the results from TEM, DLS and magnetization analyses. The DOX loading capacity was as high as $0.57 \mathrm{mg} / \mathrm{mL}$. In addition, drug release of the FA-TMLs@MNPs-GNRsDOX could be controlled by the photothermal effect through laser irradiation using a $980 \mathrm{~nm}$ laser beam on the FA-TMLs@MNPs-GNRs-DOX to trigger structural changes of the FA-TMLs. An average of $95 \%$ of the drug was released after 3 hours. The results of cell uptake studies indicate that FA-TMLs@MNPs-GNRs-DOX were able to specifically bind folate-receptor-positive cells and showed significant toxicity to bladder tumor cells. All of the aforementioned results demonstrate that the present FATMLs@MNPs-GNRs-DOX system has promising prospects for use as a multi-functionalized drug delivery system for bladder tumor therapy. However, further studies are needed to verify the efficacy of multistage targeting mode for bladder cancer in vivo. The nanocarriers were first targeted to the lesions detected by CT by magnetic targeting, and then preferentially entered bladder cancer cells by folic acid targeting. Finally, NIR irradiation was used to release doxorubicin loaded with nanocarrier, and chemotherapy and photothermal physiotherapy were used to complete cancer treatment. The potential problems in the process are the need to optimize magnetic field intensity, laser intensity and time, drug concentration and other parameters, in order to obtain a good therapeutic effect.

\section{Acknowledgments}

We gratefully acknowledge the support from the Jiangsu specially appointed professor program, the Science and Technology Project (Social Development) of Wujin District (WS201809) and Changzhou Health and Family Planning Commission Youth Foundation of China (QN201814).

\section{Author Contributions}

All authors made a significant contribution to the work reported, whether that is in the conception, study design, execution, acquisition of data, analysis and interpretation, or in all these areas; took part in drafting, revising or critically reviewing the article; gave final approval of the version to be published; have agreed on the journal to which the article has been submitted; and agree to be accountable for all aspects of the work.

\section{Disclosure}

The authors declare no conflicts of interest for this work.

\section{References}

1. Siegel RL, Miller KD, Jemal A. Cancer statistics, 2019. CA Cancer J Clin. 2019;69(1):7-34. doi:10.3322/caac.21551

2. Chamie K, Litwin MS, Bassett JC, et al. Recurrence of high-risk bladder cancer: a population-based analysis. Cancer. 2013;119 (17):3219-3227. doi:10.1002/cncr.28147

3. Kaufman DS, Shipley WU, Feldman AS. Bladder cancer. The Lancet. 2009;374(9685):239-249. doi:10.1016/S0140-6736(09)60491-8

4. Kirkali Z, Chan T, Manoharan M, et al. Bladder cancer: epidemiology, staging and grading, and diagnosis. Urology. 2005;66(6 Suppl 1):4-34. doi:10.1016/j.urology.2005.07.062

5. Shen Z, Shen T, Wientjes MG, O'Donnell MA, Au JL. Intravesical treatments of bladder cancer: review. Pharm Res. 2008;25 (7):1500-1510. doi:10.1007/s11095-008-9566-7

6. Kolawole OM, Lau WM, Mostafid H, Khutoryanskiy VV. Advances in intravesical drug delivery systems to treat bladder cancer. Int J Pharm. 2017;532(1):105-117. doi:10.1016/j.ijpharm.2017.08.120

7. Tibbitt MW, Dahlman JE, Langer R. Emerging Frontiers in Drug Delivery. J Am Chem Soc. 2016;138(3):704-717.

8. Douglass L, Schoenberg M. The Future of Intravesical Drug Delivery for Non-Muscle Invasive Bladder Cancer. Bladder Cancer. 2016;2 (3):285-292.

9. Jaiswal MK, Pradhan L, Vasavada S, et al. Magneto-thermally responsive hydrogels for bladder cancer treatment: therapeutic efficacy and in vivo biodistribution. Colloids Surf $B$ Biointerfaces. 2015;136:625-633. 
10. Guo H, Xu W, Chen J, et al. Positively charged polypeptide nanogel enhances mucoadhesion and penetrability of 10-hydroxycamptothecin in orthotopic bladder carcinoma. J Control Release. 2017;259:136-148. doi:10.1016/j.jconrel.2016.12.041

11. Huang Z, Xiao H, Lu X, Yan W, Ji Z. Enhanced photo/chemo combination efficiency against bladder tumor by encapsulation of DOX and $\mathrm{ZnPC}$ into in situ-formed thermosensitive polymer hydrogel. Int $J$ Nanomedicine. 2018;13:7623-7631. doi:10.2147/ IJN.S179226

12. Dan P, Jeffrey MK, SeungPyo H. Nanocarriers as an emerging platform for cancer therapy. Nat Nanotechnol. 2007;2:751-760. doi:10.1038/nnano.2007.387

13. Hillaireau H, Couvreur P. Nanocarriers' entry into the cell: relevance to drug delivery. Cell Mol Life Sci. 2009;66(17):2873-2896. doi:10.1007/s00018-009-0053-z

14. Panikar SS, Banu N, Haramati J, Toro-Arreola DS, Leal AR, Salas P. Nanobodies as efficient drug-carriers: progress and trends in chemotherapy. $J$ Controlled Release. 2021;334:389-412. doi:10.1016/j.jconrel.2021.05.004

15. Yu K, Liu M, Dai H, Huang X. Targeted drug delivery systems for bladder cancer therapy. J Drug Deliv Sci Technol. 2020;5:56.

16. Zacche MM, Srikrishna S, Cardozo L. Novel targeted bladder drug-delivery systems: a review. Res Rep Urol. 2015;7:169-178. doi:10.2147/RRU.S56168

17. Lin X, Li L, Li S, et al. Targeting the Opening of Mitochondrial Permeability Transition Pores Potentiates Nanoparticle Drug Delivery and Mitigates Cancer Metastasis. Adv Sci. 2021;8 (4):2002834. doi:10.1002/advs.202002834

18. Brigger I, Dubernet C, Couvreur P. Nanoparticles in cancer therapy and diagnosis. Adv Drug Deliv Rev. 2002;54:631-651. doi:10.1016/ S0169-409X(02)00044-3

19. Sun T, Zhang YS, Pang B, Hyun DC, Yang M, Xia Y. Engineered nanoparticles for drug delivery in cancer therapy. Angew Chem Int Ed Engl. 2014;53(46):12320-12364. doi:10.1002/anie.201403036

20. Gao H, Bi Y, Wang X, et al. Near-Infrared Guided Thermal-Responsive Nanomedicine against Orthotopic Superficial Bladder Cancer. ACS Biomater Sci Eng. 2017;3(12):3628-3634. doi:10.1021/acsbiomaterials.7b00405

21. Gao H, Bi Y, Chen J, et al. Near-Infrared Light-Triggered Switchable Nanoparticles for Targeted Chemo/Photothermal Cancer Therapy. ACS Appl Mater Interfaces. 2016;8(24):15103-15112. doi:10.1021/ acsami.6b03905

22. Guo H, Li F, Xu W, et al. Mucoadhesive Cationic Polypeptide Nanogel with Enhanced Penetration for Efficient Intravesical Chemotherapy of Bladder Cancer. Adv Sci. 2018;5(6):1800004. doi:10.1002/advs.201800004

23. Choi H, Cho SH, Hahn SK. Urease-Powered Polydopamine Nanomotors for Intravesical Therapy of Bladder Diseases. ACS Nano. 2020;14(6):6683-6692. doi:10.1021/acsnano.9b09726

24. Wei Y, Gao L, Wang L, et al. Polydopamine and peptide decorated doxorubicin-loaded mesoporous silica nanoparticles as a targeted drug delivery system for bladder cancer therapy. Drug Deliv. 2017;24(1):681-691. doi:10.1080/10717544.2017.1309475

25. Ye QN, Wang Y, Shen S, Xu CF, Wang J. Biomaterials-Based Delivery of Therapeutic Antibodies for Cancer Therapy. $A d v$ Healthc Mater. 2021;10:e2002139. doi:10.1002/adhm.202002139

26. Arslan FB, Ozturk Atar K, Calis S. Antibody-mediated drug delivery Int J Pharm. 2021;596:120268. doi:10.1016/j.ijpharm.2021.120268

27. Sudimack J, Lee RJ. Targeted drug delivery via the folate receptor. $A d v$ Drug Deliv Rev. 2000;41:147-162. doi:10.1016/S0169-409X(99)00062-9

28. Weitman SD, Lark RH, Coney LR, et al. Distribution of the Folate Receptor GP38 in Normal and Malignant Cell Lines and Tissues. Cancer Res. 1992;52:3396-3401.

29. Dhawan D, Ramos-Vara JA, Naughton JF, et al. Targeting folate receptors to treat invasive urinary bladder cancer. Cancer Res. 2013;73(2):875-884. doi:10.1158/0008-5472.CAN-12-2101
30. Guo M, Yan Y, Zhang $\mathrm{H}$, et al. Magnetic and $\mathrm{pH}$-responsive nanocarriers with multilayer core-shell architecture for anticancer drug delivery. J Mater Chem. 2008;18:42. doi:10.1039/ b810061f

31. Fortes Brollo ME, Dominguez-Bajo A, Tabero A, et al. Combined magnetoliposome formation and drug loading in one step for efficient alternating current-magnetic field remote-controlled drug release. ACS Appl Mater Interfaces. 2020;12(4):4295-4307. doi:10.1021/ acsami.9b20603

32. Marie H, Lemaire L, Franconi F, et al. Superparamagnetic liposomes for MRI monitoring and external magnetic field-induced selective targeting of malignant brain tumors. Adv Funct Mater. 2015;25 (8):1258-1269. doi:10.1002/adfm.201402289

33. Dwivedi P, Kiran S, Han S, et al. Magnetic targeting and ultrasound activation of liposome-microbubble conjugate for enhanced delivery of anticancer therapies. ACS Appl Mater Interfaces. 2020;12 (21):23737-23751. doi:10.1021/acsami.0c05308

34. Lu Y, Chuang E, Cheng Y, Anilkumar TS, Chen H, Chen J. Thermosensitive magnetic liposomes for alternating magnetic field-inducible drug delivery in dual targeted brain tumor chemotherapy. Chem Eng J. 2019;373:720-733. doi:10.1016/j. cej.2019.05.055

35. Colombo M, Carregal-Romero S, Casula MF, et al. Biological applications of magnetic nanoparticles. Chem Soc Rev. 2012;41 (11):4306-4334. doi:10.1039/c2cs15337h

36. Hsu H, Chen J. Preparation of thermosensitive magnetic liposome encapsulated recombinant tissue plasminogen activator for targeted thrombolysis. J Magn Magn Mater. 2017;427:188-194. doi:10.1016/ j.jmmm.2016.10.122

37. Huang L, He M, Chen B, Hu B. A designable magnetic MOF composite and facile coordination-based post-synthetic strategy for the enhanced removal of $\mathrm{Hg} 2+$ from water. J Mater Chem A. 2015;3 (21):11587-11595. doi:10.1039/C5TA01484K

38. Nikoobakht B, El-Sayed MA. Preparation and growth mechanism of gold nanorods (NRs) using seed-mediated growth method. Chem Mater. 2003;15:1957-1962. doi:10.1021/cm0207321

39. Lv SW, Liu Y, Xie M, et al. Near-infrared light-responsive hydrogel for specific recognition and photothermal site-release of circulating tumor cells. ACS Nano. 2016;10(6):6201-6210. doi:10.1021/ acsnano.6b02208

40. Ke X, Lin W, Li X, Wang H, Xiao X, Guo Z. Synergistic dual-modified liposome improves targeting and therapeutic efficacy of bone metastasis from breast cancer. Drug Deliv. 2017;24 (1):1680-1689. doi:10.1080/10717544.2017.1396384

41. Dong Z, Feng L, Chao Y, et al. Amplification of tumor oxidative stresses with liposomal fenton catalyst and glutathione inhibitor for enhanced cancer chemotherapy and radiotherapy. Nano Lett. 2019;19 (2):805-815. doi:10.1021/acs.nanolett.8b03905

42. Feng W, Han $X$, Wang R, et al. Nanocatalysts-augmented and photothermal-enhanced tumor-specific sequential nanocatalytic therapy in both NIR-I and NIR-II biowindows. Adv Mater. 2019;31(5): e1805919. doi:10.1002/adma.201805919

43. Meng L, Chen W, Tan Y, et al. Fe3O4 octahedral colloidal crystals. Nano Res. 2011;4(4):370-375. doi:10.1007/s12274-010-0091-8

44. Sun J, Zhou S, Hou P, et al. Synthesis and characterization of biocompatible $\mathrm{Fe} 3 \mathrm{O} 4$ nanoparticles. $J$ Biomed Mater Res A. 2007;80(2):333-341. doi:10.1002/jbm.a.30909

45. Hua M, Yang H, Liu H, et al. Superhigh-magnetization nanocarrier as a doxorubicin delivery platform for magnetic targeting therapy. Biomaterials. 2011;32(34):8999-9010. doi:10.1016/j.biomaterials. 2011.08.014

46. Rengan AK, Jagtap M, De A, Banerjee R, Srivastava R. Multifunctional gold coated thermo-sensitive liposomes for multimodal imaging and photo-thermal therapy of breast cancer cells. Nanoscale. 2014;6(2):916-923. doi:10.1039/C3NR04448C 
47. Chen $\mathrm{C}, \mathrm{Hu} \mathrm{J}$, Zeng $\mathrm{P}$, et al. Molecular mechanisms of anticancer action and cell selectivity of short alpha-helical peptides. Biomaterials. 2014;35(5):1552-1561. doi:10.1016/j.biomaterials.2013.10.082

48. Allen TM, Cullis PR. Liposomal drug delivery systems: from concept to clinical applications. Adv Drug Deliv Rev. 2013;65(1):36-48. doi:10.1016/j.addr.2012.09.037

49. Bozzuto G, Molinari A. Liposomes as nanomedical devices. Int J Nanomedicine. 2015;10:975-999. doi:10.2147/IJN.S68861

50. Safinya CR, Ewert KK. Liposomes derived from molecular vases. Nature. 2012;489:372-374. doi:10.1038/489372b

51. Barenholz Y. Doxil(R)-the first FDA-approved nano-drug: lessons learned. J Control Release. 2012;160(2):117-134. doi:10.1016/j. jconrel.2012.03.020

52. Liang PC, Chen YC, Chiang CF, et al. Doxorubicin-modified magnetic nanoparticles as a drug delivery system for magnetic resonance imaging-monitoring magnet-enhancing tumor chemotherapy. Int J Nanomedicine. 2016;11:2021-2037. doi:10.2147/IJN.S94139

53. Lal S, Clare SE, Halas NJ. Nanoshell-Enabled Photothermal Cancer Therapy: impending Clinical Impact. Acc Chem Res. 2008;41:1842-1851. doi:10.1021/ar800150g

54. Yavuz MS, Cheng Y, Chen J, et al. Gold nanocages covered by smart polymers for controlled release with near-infrared light. Nat Mater. 2009;8(12):935-939. doi:10.1038/nmat2564

55. Hu M, Chen J, Li ZY, et al. Gold nanostructures: engineering their plasmonic properties for biomedical applications. Chem Soc Rev. 2006;35(11):1084-1094. doi:10.1039/b517615h

56. Khoury CG, Vo-Dinh T. Gold nanostars for surface-enhanced Raman scattering synthesis, characterization and optimization. J Phys Chem C. 2008;112:18849-18859. doi:10.1021/jp8054747

57. Negru B, McAnally MO, Mayhew HE, et al. Fabrication of gold nanosphere oligomers for surface-enhanced femtosecond stimulated Raman spectroscopy. J Phys Chem C. 2017;121(48):27004-27008. doi:10.1021/acs.jpcc.7b09664
58. Elnakat H, Ratnam M. Distribution, functionality and gene regulation of folate receptor isoforms: implications in targeted therapy. $A d v$ Drug Deliv Rev. 2004;56(8):1067-1084. doi:10.1016/j. addr.2004.01.001

59. Tomeh MA, Zhao X. Recent advances in microfluidics for the preparation of drug and gene delivery systems. Mol Pharm. 2020;17 (12):4421-4434. doi:10.1021/acs.molpharmaceut.0c00913

60. Liu H, Singh RP, Zhang Z, Han X, Liu Y, Hu L. Microfluidic assembly: an innovative tool for the encapsulation, protection, and controlled release of nutraceuticals. J Agric Food Chem. 2021;69 (10):2936-2949. doi:10.1021/acs.jafc.0c05395

61. Chan KH, Tay JJJ. Advancement of peptide nanobiotechnology via emerging microfluidic technology. Micromachines. 2019;10:10.

62. Kautzka Z, Clement S, Goldys EM, Deng W. Light-triggered liposomal cargo delivery platform incorporating photosensitizers and gold nanoparticles for enhanced singlet oxygen generation and increased cytotoxicity. Int J Nanomedicine. 2017;12:969-977. doi:10.2147/IJN. S126553

63. Lv SW, Wang J, Xie M, et al. Photoresponsive immunomagnetic nanocarrier for capture and release of rare circulating tumor cells. Chem Sci. 2015;6(11):6432-6438. doi:10.1039/C5SC01380A

64. Liu MC, Liu L, Wang XR, et al. Folate receptor-targeted liposomes loaded with a diacid metabolite of norcantharidin enhance antitumor potency for H22 hepatocellular carcinoma both in vitro and in vivo. Int J Nanomedicine. 2016;11:1395-1412.

65. Xiong XB, Ma Z, Lai R, Lavasanifar A. The therapeutic response to multifunctional polymeric nano-conjugates in the targeted cellular and subcellular delivery of doxorubicin. Biomaterials. 2010;31 (4):757-768.

66. Chazotte B. Labeling lysosomes in live cells with LysoTracker. Cold Spring Harb Protoc. 2011;2011(2):pdbprot5571.
International Journal of Nanomedicine

\section{Publish your work in this journal}

The International Journal of Nanomedicine is an international, peerreviewed journal focusing on the application of nanotechnology in diagnostics, therapeutics, and drug delivery systems throughout the biomedical field. This journal is indexed on PubMed Central, MedLine, CAS, SciSearch ${ }^{\mathbb{}}$, Current Contents ${ }^{\mathbb{R}} /$ Clinical Medicine, $^{-}$

\section{Dovepress}

Journal Citation Reports/Science Edition, EMBase, Scopus and the Elsevier Bibliographic databases. The manuscript management system is completely online and includes a very quick and fair peer-review system, which is all easy to use. Visit http://www.dovepress.com/ testimonials.php to read real quotes from published authors. 